\title{
Achievable Rate of Multi-Relay Cognitive Radio MIMO Channel with Space Alignment
}

\author{
Lokman Sboui, Hakim Ghazzai, Zouheir Rezki, and Mohamed-Slim Alouini \\ Computer, Electrical and Mathematical Sciences and Engineering (CEMSE) Division \\ King Abdullah University of Science and Technology (KAUST) \\ Thuwal, Makkah Province, Saudi Arabia \\ \{lokman.sboui, hakim.ghazzai, zouheir.rezki, slim.alouini\}@kaust.edu.sa
}

\begin{abstract}
We study the impact of multiple relays on the primary user (PU) and secondary user (SU) rates of underlay MIMO cognitive radio. Both users exploit amplify-and-forward relays to communicate with the destination. A space alignment technique and a special linear precoding and decoding scheme are applied to allow the $\mathrm{SU}$ to use the resulting free eigenmodes. In addition, the $\mathrm{SU}$ can communicate over the used eigenmodes under the condition of respecting an interference constraint tolerated by the PU. At the destination, a successive interference cancellation (SIC) is performed to estimate the secondary signal. We present the explicit expressions of the optimal PU and SU powers that maximize their achievable rates. In the numerical results, we show that our scheme provides cognitive rate gain even in absence of tolerated interference. In addition, we show that increasing the number of relays enhances the $P U$ and $S U$ rates at low power regime and/or when the relays power is sufficiently high.
\end{abstract}

Index Terms-Underlay cognitive radio, MIMO space alignment, amplify-and-forward multiple-relay.

\section{INTRODUCTION}

In order to cope with the continuous growth of wireless networks, new emerging systems need to offer higher data rate and to overcome bandwidth shortage. Consequently, many techniques have been presented to enhance the network performances and spectrum scarcity [1]. From one side, the cognitive radio (CR) paradigm was introduced to avoid spectrum inefficient allocation. In this paradigm, unlicensed secondary users (SU's) are allowed to share the spectrum with licensed primary users (PU's) under the condition of maintaining the PU quality of service (QoS). One of the CR modes is the underlay mode in which the PU tolerates a certain level of interference coming from the SU [2]. From the other side, relay-assisted communications [3], was introduced as a solution to considerably enhance distant and non-line of sight communications. The relying was first intended to enhance single-antenna communications. Nevertheless, relaying in MIMO systems was shown to improve performances as well [4]. In addition, adopting MIMO power allocation within a CR framework has been studied previously in, e.g., [5][7]. In [5], MIMO space alignment was adopted but without relaying. In [6], the space alignment (SA) technique was introduced to mitigate $\mathrm{SU}$ interference by exploiting the free eigenmodes of MIMO systems. In [7], the authors present the $\mathrm{CR}$ rate after optimizing the power under interference and budget power constraints. From another side, CR with multi-relays networks was studied in [8]. To the best of our knowledge, sharing multiple-relays with the PU in a CR setting was not studied before. In [8], the authors only consider the SU transmission and respecting only interference constraints. In [9], the multiple-relays CR with interference constraint was studied. However, the analyzed performance metric was the outage probability. In [10], the authors consider a multiple relay CR without considering the PU. In addition, only the interference from the relays is considered, and the SU interference was not analyzed. From another side, communicating to the same destination in CR context was studied in previously, i.e. [11]-[13], but with no multiple-relaying.

In this paper, we study a multi-relay CR system with a proposed linear precoding and decoding that simplify the derivation of the optimal power. Our objective is to maximize the achievable rate of both the primary and the cognitive users, as well as the effect of the number of relays on these rates. The motivation of this study is to investigate the eventual gain of the cognitive users when sharing, in addition to the spectrum, the multiple relays with the PU's. Hence, we are interested in analyzing the effect of the number of relays on the rates. In our setting, after a particular precoding at the PU transmitter, the set of PU-relays channels is transformed into parallel channels with some free eigenmodes that can be freely exploited by the SU. Nevertheless, the SU also transmits through the used eigenmodes but respecting an interference constraint tolerated by the PU. At the destination, the PU and the SU signals are decoded using a Successive Interference Cancellation (SIC) decoder [14].

The rest of this paper is organized as follows. In Section II, the system model is presented. Section III describes the PU precoding under space alignment. SU achievable rate expressions are derived for various SIC accuracies in Section IV. Numerical results are presented in Section V. Finally, the paper is concluded in Section VI.

\section{SySTEM MOdEL}

In our system model, we study an uplink communication scenario as depicted in Fig.1 where the "PU" and the "SU" are transmitting their signals simultaneously to a common destination " $D$ ". The destination could be seen as a base station to which the SU is trying to communicate under the underlay CR concept. We assume that there is no direct link between 


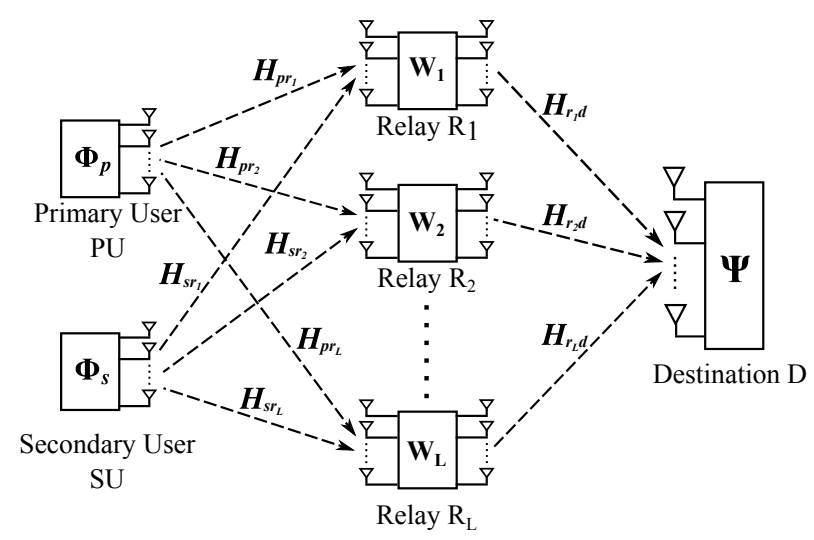

Figure 1: Uplink spectrum sharing communication in the presence of a multiple relays.

the transmitters and the common receiver. Instead, there are $L$ relays, $R_{1}, \cdots, R_{L}$, that can receive and amplify the PU and SU signals and forward the amplified to the destination D. As a licensed user, the PU is free to exploit the channel. Meanwhile, the SU, as an unlicensed user, can share opportunistically the spectrum under some constraints that preserve a certain Quality of Service (QoS) of the PU communication. We assume that each node has $N$ antennas, and the channel gain matrices representing the links between the $\mathrm{PU}$ and $\mathrm{R}_{l}\left(\mathrm{PU}-\mathrm{R}_{l}\right)$ between SU and $\mathrm{R}_{l}\left(\mathrm{SU}-\mathrm{R}_{l}\right)$, and between $\mathrm{R}_{l}$ and $\mathrm{D}\left(\mathrm{R}_{l}-\mathrm{D}\right)$ are denoted by $\boldsymbol{H}_{\boldsymbol{p r _ { l }}}, \boldsymbol{H}_{\boldsymbol{s r}_{l}}$, and $\boldsymbol{H}_{\boldsymbol{r} \boldsymbol{d}_{l}}$, respectively, $l=1, \ldots, L$.. All channel matrices are assumed to be independent. In the first time slot, the transmitters transmit simultaneously their signal to the relays where the complex received vector at each relay $R_{l}, l=1, \ldots, L$, is given by:

$$
\boldsymbol{y}_{R_{l}}=\boldsymbol{H}_{p r_{l}} \boldsymbol{\Phi}_{\boldsymbol{p}} \boldsymbol{s}_{\boldsymbol{p}}+\boldsymbol{H}_{s r_{l}} \boldsymbol{\Phi}_{\boldsymbol{s}} \boldsymbol{s}_{\boldsymbol{s}}+\boldsymbol{z}_{R_{l}},
$$

where $\boldsymbol{\Phi}_{p}$ and $\boldsymbol{\Phi}_{s}$ are the linear precoding matrices applied at the PU and $\mathrm{SU}$, and $s_{p}$ and $s_{s}$ are the transmitted signals by PU and SU, respectively, assumed to be independent and identically distributed (i.i.d) complex Gaussian. The covariance matrix of the vector $\boldsymbol{s}_{\boldsymbol{i}}, i \in\{p, s\}$, are $\boldsymbol{P}_{\boldsymbol{i}}=\mathbb{E}\left[\boldsymbol{s}_{\boldsymbol{i}} \boldsymbol{s}_{\boldsymbol{i}}{ }^{h}\right]$, where $\mathbb{E}[\cdot]$ is the expectation operator over all channel realizations and.$^{h}$ is the transpose conjugate operator. This covariance matrix is constrained by a power constraint $\operatorname{Tr}\left(\boldsymbol{\Phi}_{\boldsymbol{i}} \boldsymbol{P}_{\boldsymbol{i}} \boldsymbol{\Phi}_{\boldsymbol{i}}{ }^{h}\right) \leq P_{\text {tot }}$ where $\operatorname{Tr}(\boldsymbol{A})=\sum_{j} A(j, j)$ is the trace of the matrix $\boldsymbol{A}$, and $P_{t o t}$ is the total power budget considered, without loss of generality, to be the same for both users. The noise $\boldsymbol{z}_{R_{l}}, l=1, \ldots, L$, is a zero mean additive white Gaussian noise (AWGN) vector at the relay $R_{l}, l=$ $1, \ldots, L$, with an identity covariance matrix, $\boldsymbol{I}_{\boldsymbol{N}}$.

In the second time slot, each relay $R_{l}, l=1, \ldots, L$, amplifies the signal $\boldsymbol{y}_{\boldsymbol{R}_{l}}$ through a gain matrix denoted $\boldsymbol{W}_{\boldsymbol{l}}$ before retransmitting the signal to $\mathrm{D}$. We denote by $P_{R_{l}}$ the budget power of each relay $R_{l}$. The total received signal $\boldsymbol{y}_{D}$ at the receiver $\mathrm{D}$ can be written as follows

$$
y_{D}=H_{p d} \Phi_{p} s_{p}+H_{s d} \Phi_{s} s_{s}+z
$$

where $\boldsymbol{H}_{\boldsymbol{p d}}=\sum_{l=1}^{L} \boldsymbol{H}_{\boldsymbol{r}_{l} \boldsymbol{d}} \boldsymbol{W}_{\boldsymbol{l}} \boldsymbol{H}_{\boldsymbol{p} \boldsymbol{r}_{l}}, \quad \boldsymbol{H}_{\boldsymbol{s d}}=$ $\sum_{l=1}^{L} \boldsymbol{H}_{\boldsymbol{r}_{\boldsymbol{l}} \boldsymbol{d}} \boldsymbol{W}_{\boldsymbol{l}} \boldsymbol{H}_{\boldsymbol{s} \boldsymbol{r}_{\boldsymbol{l}}} \quad$ and $\boldsymbol{z}=\sum_{l=1}^{L} \boldsymbol{H}_{\boldsymbol{r}_{\boldsymbol{l}} \boldsymbol{d}} \boldsymbol{W}_{\boldsymbol{l}} \boldsymbol{z}_{\boldsymbol{R}_{\boldsymbol{l}}}+\boldsymbol{z}_{\boldsymbol{D}}$.
The noise $z_{D}$ is a AWGN vector at the destination D with an identity covariance matrix, $\boldsymbol{I}_{\boldsymbol{N}}$. Consequently, the link between the PU and the D is transformed to a single channel matrix gain, $\boldsymbol{H}_{\boldsymbol{p d}}$ involving all the $2 \times L$ channel gain matrices that link the L relays with the PU and the D. The same transformation is applied to the SU-D link as well and consequently the problem complexity is reduced. Note that this method can be applicable since the gain matrix at the relays are fixed and known. In case we need to optimize theses matrices, a different transformation should be adopted, e.g., matched filter [15]. We assume that full channel state information (CSI) at the transmitters, at the relays and at the receiver. Note that when a common receiver is considered, the PU and SU signals are subject to a mutual interference that may affect both PU and SU performances. Therefore, we adopt an interference constraint [16], in order to protect the licensed PU. This constraint is imposed by the PU on the SU transmission to be below a certain interference threshold per receive antenna denoted by $I_{t h}$.

\section{Primary USer Precoding With SPACE ALIGNMENT}

We propose a linear precoding and decoding matrices used to maximize the both $\mathrm{PU}$ and $\mathrm{SU}$ rates while respecting the PU's QoS. In this scheme the space alignment technique [6] is adopted. This technique allows the SU to transmit through the unused primary eigenmodes. By having perfect CSI as well as the relay gain matrices, the PU performs an optimal power allocation that maximizes its rate by applying a Singular Value Decomposition (SVD) to $\boldsymbol{H}_{\boldsymbol{p d}}$ denoted $\boldsymbol{H}_{\boldsymbol{p d}}=\boldsymbol{U} \boldsymbol{\Lambda} \boldsymbol{V}^{h}$ where $\boldsymbol{U}$ and $\boldsymbol{V}$ are two unitary matrices and $\boldsymbol{\Lambda}$ is a diagonal matrix that contains the ordered singular values of $\boldsymbol{H}_{\boldsymbol{p d}}$ denoted as $\lambda_{1} \geq \lambda_{2} \geq \cdots \geq \lambda_{N}$. Thus, the PU transmits through parallel channels associated to their eigenmodes. Afterwards, in order to transform the PU channel to $N$ parallel channels, we employ the linear precoding at the PU transmitter $\boldsymbol{\Phi}_{\boldsymbol{p}}$ such as $\boldsymbol{\Phi}_{\boldsymbol{p}}=\boldsymbol{V}$ and the decoding $\boldsymbol{\Psi}$ at the destination such as $\boldsymbol{\Psi}=\boldsymbol{U}$. Consequently, the received signal after decoding is given by

$$
\boldsymbol{r}=\boldsymbol{\Psi}^{h} \boldsymbol{y}_{\boldsymbol{D}}=\boldsymbol{\Lambda} \boldsymbol{s}_{\boldsymbol{p}}+\boldsymbol{U}^{h} \boldsymbol{H}_{\boldsymbol{s d}} \boldsymbol{\Phi}_{\boldsymbol{s}} \boldsymbol{s}_{\boldsymbol{s}}+\tilde{\boldsymbol{z}}
$$

where $\tilde{\boldsymbol{z}}=\boldsymbol{U}^{h} \boldsymbol{z}$ is a zero mean AWGN with a $N$-by- $N$ covariance matrix $\boldsymbol{Q}_{\tilde{z}}=\boldsymbol{I}_{\boldsymbol{N}}+\boldsymbol{U}^{h} \boldsymbol{H}_{\boldsymbol{r d}} \boldsymbol{W} \boldsymbol{W}^{h} \boldsymbol{H}_{\boldsymbol{r d}}{ }^{h} \boldsymbol{U}$.

Meanwhile, the PU communication is protected by forcing the coming interference to be below a certain threshold denoted $I_{t h}$. Let $s$ be the received signal related to the SU transmission, i.e., $\boldsymbol{s}=\boldsymbol{U}^{h} \boldsymbol{H}_{\boldsymbol{s} \boldsymbol{d}} \boldsymbol{\Phi}_{\boldsymbol{s}} \boldsymbol{s}_{\boldsymbol{s}}$. Let also $\boldsymbol{Q}_{\boldsymbol{s}}$ to be its covariance matrix. Respecting the interference constraint means that, for each antenna $j, j=1, \ldots, N$, we have $Q_{s}(j, j) \leq I_{t h}$. In our study, the PU considers the SU interference to be $I_{t h}$ in each antenna so that the power allocation is performed. This study presents a lower bound of the PU performance since the threshold $I_{t h}$ is not always reached by the SU. The PU rate expression can be written as

$$
R_{p}=\log _{2}\left(\operatorname{det}\left[\boldsymbol{I}_{\boldsymbol{N}}+\left(\boldsymbol{\Lambda} \boldsymbol{s}_{\boldsymbol{p}}\right)\left(\boldsymbol{\Lambda} \boldsymbol{s}_{\boldsymbol{p}}\right)^{h}\left(I_{t h} \boldsymbol{I}_{\boldsymbol{N}}+\boldsymbol{Q}_{\tilde{\boldsymbol{z}}}\right)^{-1}\right]\right) \text {, }
$$


where $\operatorname{det}[\cdot]$ is the determinant operator. Since all the matrices are diagonal, this rate can be simply written as

$$
R_{p}=\sum_{j=1}^{N} \log _{2}\left(1+\frac{P_{p}(j, j) \lambda_{j}^{2}}{I_{t h}+Q_{\tilde{z}}(j, j)}\right) .
$$

Meanwhile, the PU power must respect two types of constraints: budget power constraint and the relays constraints. As mentioned in Section II, the budget power constraint is written as $\operatorname{Tr}\left(\boldsymbol{\Phi}_{p} \boldsymbol{P}_{\boldsymbol{p}} \boldsymbol{\Phi}_{p}{ }^{h}\right) \leq P_{t o t}$. By using the invariance of the Trace operator under the cyclic permutation and the unitarity of the matrix $\boldsymbol{\Phi}_{\boldsymbol{p}}$, this constraint becomes $\operatorname{Tr}\left(\boldsymbol{P}_{\boldsymbol{p}}\right) \leq P_{t o t}$. From another side, the relays constraints reflect the fact that for a given relay, $R_{l}, l=1, \ldots, L$, the transmit a signal power cannot exceed its own budget $P_{R_{l}}$ which can be written as:

$$
\operatorname{Tr}\left(\boldsymbol{W}_{\boldsymbol{l}} y_{R_{l}}\left(\boldsymbol{W}_{\boldsymbol{l}} y_{R_{l}}\right)^{h}\right) \leq P_{R_{l}} .
$$

However, since the SU must respect the interference threshold, the SU power is considered to be this threshold so that the PU can allocate its power without the need to know the exact SU power. Then, the PU actual achieved rate is greater or equal to this lower bound and is mainly derived by considering the actual SU interference instead of $I_{t h}$. By denoting $\boldsymbol{H}_{p_{l}}=$ $\boldsymbol{W}_{l} \boldsymbol{H}_{p r_{l}} \boldsymbol{\Phi}_{\boldsymbol{p}}$ and $\boldsymbol{H}_{s_{l}}=\boldsymbol{W}_{\boldsymbol{l}} \boldsymbol{H}_{s r_{l}} \boldsymbol{\Phi}_{\boldsymbol{s}}$, the optimal PU power and the rate lower

$$
\begin{aligned}
& \underset{\boldsymbol{P}_{\boldsymbol{p}}}{\operatorname{maximize}} R_{p}=\sum_{j=1}^{N} \log _{2}\left(1+\frac{P_{p}(j, j) \lambda_{j}^{2}}{I_{t h}+Q_{\tilde{z}}(j, j)}\right) \\
& \text { s.t. } \operatorname{Tr}\left(\boldsymbol{P}_{\boldsymbol{p}}\right) \leq P_{t o t}, \\
& \quad \operatorname{Tr}\left(\boldsymbol{H}_{\boldsymbol{p}_{\boldsymbol{l}}} \boldsymbol{P}_{\boldsymbol{p}} \boldsymbol{H}_{\boldsymbol{p}_{\boldsymbol{l}}}{ }^{h}+I_{t h} \boldsymbol{H}_{\boldsymbol{s}_{\boldsymbol{l}}} \boldsymbol{H}_{\boldsymbol{s}_{\boldsymbol{l}}}{ }^{h}\right. \\
& \left.\quad+\boldsymbol{W}_{\boldsymbol{l}} \boldsymbol{W}_{\boldsymbol{l}}{ }^{h}\right) \leq P_{R_{l}}, \forall l=1, \ldots, L,
\end{aligned}
$$

Since the objective function (7) is convex and the constraints are linear, this optimization problem is convex [17]. Consequently, we use the Lagrangian method to solve this problem. We first compute the Lagrangian function and then find its derivative with regards to each $P_{p}(j, j)$. The optimal power is given such as the derivative is equal to zero and is given, $\forall j=1, \ldots, N$, by:

$$
\begin{array}{r}
P_{p}^{*}(j, j)=\left[\frac{1}{\mu_{p}+\sum_{l=1}^{L}\left(\eta_{p_{l}} \sum_{i=1}^{N}\left|H_{p_{l}}(j, i)\right|^{2}\right)}\right. \\
\left.-\frac{I_{t h}+Q_{\tilde{z}}(j, j)}{\lambda_{j}{ }^{2}}\right]^{+},
\end{array}
$$

where $[.]^{+}=\max (0,.) . \mu_{p}$ and $\eta_{p_{l}}, l=1, \ldots, L$, are the Lagrangian multipliers corresponding to the power budget constraint and the relays power constraints expressed in (8) and (9), respectively. The optimal power allocation in (10) is similar to the water-filling power expression. Note also that when the channel gain is low, i.e., $\lambda_{j}$ 's have small values, the $\mathrm{PU}$ is using fewer eigenmodes than the number of antennas $N$ which gives the opportunity to the SU to exploit more free eigenmodes.

\section{Secondary User Achievable Rate}

In this section, we investigate the achievable rate of SU using the proposed strategy described in Section III depending on the SIC performance. First, we derive the SU optimal power allocation assuming a perfect SIC (a sort of genie SIC). Then, we investigate the gain in performance with an imperfect SIC (i.e., totally erroneous SIC). We introduce a parameter $\alpha$ $(0 \leq \alpha \leq 1)$ that corresponds to the probability of detecting the PU signal $s_{p}$ correctly before applying the SIC. Let $n$ $(0 \leq n<N)$ be the number of unused eigenmodes. Then, there are $N-n$ eigenmodes used by the PU and $n$ unused eigenmodes that can be freely exploited by the SU. In order to totally eliminate the effect of interference, an appropriate choice of $\boldsymbol{\Phi}_{s}$ has been proposed in [5] for a Line-of-Sight channel without relaying scheme where the SU is allowed to transmit in all the eigenmodes by respecting a certain interference temperature threshold $I_{t h}$ when sharing the used eigenmodes. $\left(\boldsymbol{H}_{\boldsymbol{s} \boldsymbol{d}}\right)^{-1} \boldsymbol{U} \overline{\boldsymbol{P}}_{\boldsymbol{p}}$, where $\overline{\boldsymbol{P}}_{\boldsymbol{p}}$ is a diagonal matrix with the following entries:

$$
\bar{P}_{p}(j, j)=\left\{\begin{array}{cc}
1 & \text { if } P_{p}(j, j)=0 \\
0 & \text { if } P_{p}(j, j) \neq 0,
\end{array} \text { for } j=1 \ldots N\right.
$$

In order to allow the SU to transmit in all the eigenmodes by respecting a certain interference temperature threshold $I_{t h}$ when sharing the used eigenmodes, we choose $\boldsymbol{\Phi}_{s}$ as follows:

$$
\boldsymbol{\Phi}_{\boldsymbol{s}}=\left(\boldsymbol{H}_{\boldsymbol{s} \boldsymbol{d}}\right)^{-1} \boldsymbol{U}
$$

without loss of generality we assume that $\boldsymbol{H}_{\boldsymbol{s} \boldsymbol{d}}$ is invertible otherwise $\left(\boldsymbol{H}_{\boldsymbol{s} \boldsymbol{d}}\right)^{-1}$ can be taken as the pseudo-inverse of $\boldsymbol{H}_{\boldsymbol{s} \boldsymbol{d}}$. In addition, since the SU knows the PU CSI and the relay gain matrices, (i.e., $\boldsymbol{H}_{\boldsymbol{p r} r_{l}}, \boldsymbol{H}_{r_{l} d}$ and $\boldsymbol{W}_{l}$ ), the unitary matrix $\boldsymbol{U}$ can be computed at the SU transmitter. We assume here that there is a feedback through which the receiver can broadcast this information to the cognitive user. Consequently,

the received signal at the $\mathrm{D}$ is expressed in the two following sets depending on the number of free eigenmodes

$$
\begin{aligned}
& r_{D j}=\lambda_{j} s_{p_{j}}+s_{s j}+\tilde{z}_{j}, \forall j=1, \ldots, N-n, \\
& r_{D j}=s_{s j}+\tilde{z}_{j}, \forall j=N-n+1, \ldots, N .
\end{aligned}
$$

Since the SU power is constrained by $I_{t h}$, a SIC is performed at the D to decode the SU signal and to remove the effect $s_{p}$ from the received signal. Meanwhile, the SU signal transmitted over the $n$ free eigenmodes is only constrained by the budget and relays constraints.

\section{A. Perfect SIC}

A perfect SIC is reached when the PU signal is always decoded perfectly, i.e., $\hat{s}_{p_{j}}=s_{p_{j}}, \forall j=1, \ldots, N-n$, where $\hat{s}_{p_{j}}$ is the estimated PU signal at the $\mathrm{j}^{\text {th }}$ receive antenna. Consequently, the cancellation of the PU effect on the SU signal is performed correctly $(\alpha=1)$ and corresponding received signal after the SIC decoding, $\tilde{r}$, is written as

$$
\tilde{r}=r-\Lambda \hat{s}_{p}=s_{s}+\tilde{z} .
$$


Hence, the corresponding SU rate is given by solving the following optimization problem

$$
\begin{aligned}
& \max _{\boldsymbol{P}_{\boldsymbol{s}}} R_{s}{ }^{(1)}=\sum_{j=1}^{N} \log _{2}\left(1+\frac{P_{s}(j, j)}{Q_{\tilde{z}}(j, j)}\right) \\
& \text { s.t. } \operatorname{Tr}\left(\boldsymbol{\Phi}_{\boldsymbol{s}} \boldsymbol{P}_{\boldsymbol{s}} \boldsymbol{\Phi}_{\boldsymbol{s}}{ }^{h}\right) \leq P_{t o t}, \\
& \quad \operatorname{Tr}\left(\boldsymbol{H}_{\boldsymbol{p}_{l}} \boldsymbol{P}_{\boldsymbol{p}}^{*} \boldsymbol{H}_{\boldsymbol{p}_{\boldsymbol{l}}}{ }^{h}+\boldsymbol{H}_{\boldsymbol{s}_{l}} \boldsymbol{P}_{\boldsymbol{s}} \boldsymbol{H}_{\boldsymbol{s}_{l}}{ }^{h}\right. \\
& \left.\quad+\boldsymbol{W}_{\boldsymbol{l}} \boldsymbol{W}_{\boldsymbol{l}}{ }^{h}\right) \leq P_{R_{l}}, \forall l=1, \ldots, L, \\
& \quad P_{s}(j, j) \leq I_{t h}, \forall j=1, \ldots, N-n,
\end{aligned}
$$

where $\boldsymbol{P}_{p}^{*}$ is the optimal PU power obtained after solving the optimization problem given in (7)-(9). The problem (15)-(18) is a convex problem as the objective function is convex and the constraints are linear. The constraint (16) can be written as $\operatorname{Tr}\left(\boldsymbol{\Phi}_{\boldsymbol{s}}{ }^{h} \boldsymbol{\Phi}_{s} \boldsymbol{P}_{\boldsymbol{s}}\right) \leq P_{t o t}$ after using the invariance of the Trace operator under the cyclic permutation. Let the matrix $\boldsymbol{A}_{\boldsymbol{s}}=\boldsymbol{\Phi}_{\boldsymbol{s}}{ }^{h} \boldsymbol{\Phi}_{\boldsymbol{s}}$, then (16) becomes $\operatorname{Tr}\left(\boldsymbol{A}_{\boldsymbol{s}} \boldsymbol{P}_{\boldsymbol{s}}\right) \leq P_{\text {tot }}$. Now, since the constraint (18) is a peak constraint, we solve this problem by solving two subproblems with the same objective function. The first subproblem has the constraints (16), (17) whereas the second has the constraint (18). Afterward, the solution of the main problem is given by taking minimum between the two solutions [18]. The first subproblem is solved by using the Lagrange method [17], and an optimal solution similar to (10) is found. In the second subproblem, the optimal solution is simply $I_{t h} \forall j=1, \ldots, N-n$. Consequently, the resulting power profile is given as follows:

$$
\begin{aligned}
& P_{s}^{*}(j, j)= \\
& \left\{\begin{array}{r}
\min \left\{\left[\frac{1}{\mu_{s} A_{s}(j, j)+\sum_{l=1}^{L}\left(\eta_{s_{l}} \sum_{i=1}^{N}\left|H_{s_{l}}(j, i)\right|^{2}\right)}\right.\right. \\
\left.\left.-Q_{\tilde{z}}(j, j)\right]^{+}, I_{t h}\right\}, \forall j=1, \ldots, N-n, \\
{\left[\frac{1}{\mu_{s} A_{s}(j, j)+\sum_{l=1}^{L}\left(\eta_{s_{l}} \sum_{i=1}^{N}\left|H_{s_{l}}(j, i)\right|^{2}\right)}-Q_{\tilde{z}}(j, j)\right]^{+},} \\
\forall j=N-n+1, \ldots, N,
\end{array}\right.
\end{aligned}
$$

where $\mu_{s}$ and $\eta_{s_{l}}, l=1, \ldots, L$, are the Lagrange multipliers associated to the budget power and the relays constraints, respectively. The optimal SU power in (19) does not involve directly the PU power allocation. However, it is affected by the number of free eigenmodes. Moreover, even in the case where the PU does not tolerate any interference, i.e. $I_{t h}=0$, the $\mathrm{SU}$ is able to transmit through the free eigenmodes and the corresponding rate is called the free eigenmodes (FE) rate.

\section{B. Imperfect SIC}

We previously analyzed the case where capacity achieving codes are employed by the PU transmitter. In this subsection, instead of using capacity achieving codes, the PU uses more practical coding schemes that may lead to unavoidable decoding errors. To this extent, we have introduced the parameter $\alpha$ the represents the accuracy of the SIC. We now investigate the case of $\alpha=0$, when an imperfect SIC is employed. In this case, the interference power at each antenna is equal to $\mathbb{E}\left[\left|\tilde{\lambda}_{j}\left(s_{p_{j}}-\hat{s}_{p_{j}}\right)\right|^{2}\right]=2 P_{p}^{*}(j, j) \lambda_{j}{ }^{2}$. The corresponding SU achievable rate is obtained by solving the following optimization problem:

$$
\begin{gathered}
\max _{\boldsymbol{P}_{\boldsymbol{s}}} R_{s}{ }^{(0)}=\sum_{j=1}^{N-n} \log _{2}\left(1+\frac{P_{s}(j, j)}{Q_{\tilde{z}}(j, j)+2 P_{p}^{*}(j, j) \lambda_{j}{ }^{2}}\right) \\
\quad+\sum_{j=N-n+1}^{N} \log _{2}\left(1+\frac{P_{s}(j, j)}{Q_{\tilde{z}}(j, j)}\right) \\
\text { s.t. } \operatorname{Tr}\left(\boldsymbol{A}_{\boldsymbol{s}} \boldsymbol{P}_{\boldsymbol{s}}\right) \leq P_{t o t}, \\
\quad \operatorname{Tr}\left(\boldsymbol{H}_{\boldsymbol{p}_{l}} \boldsymbol{P}_{\boldsymbol{p}}^{*} \boldsymbol{H}_{\boldsymbol{p}_{l}}{ }^{h}+\boldsymbol{H}_{\boldsymbol{s}_{l}} \boldsymbol{P}_{\boldsymbol{s}} \boldsymbol{H}_{\boldsymbol{s}_{l}}{ }^{h}\right. \\
\left.\quad+\boldsymbol{W}_{\boldsymbol{l}} \boldsymbol{W}_{\boldsymbol{l}}{ }^{h}\right) \leq P_{R_{l}}, \forall l=1, \ldots, L \\
P_{s}(j, j) \leq I_{t h}, \forall j=1, \ldots, N-n,
\end{gathered}
$$

Using the convexity of this problem, the optimal power is computed by using the Lagrange method, similarly to the perfect SIC case. The resulting solution is

$$
\begin{aligned}
& P_{s}^{*}(j, j)= \\
& \left\{\begin{array}{c}
\min \left\{\left[\frac{1}{\mu_{s} A_{s}(j, j)+\sum_{l=1}^{L}\left(\eta_{s_{l}} \sum_{i=1}^{N}\left|H_{s_{l}}(j, i)\right|^{2}\right)}\right.\right. \\
\left.\left.-\left(Q_{\tilde{z}}(j, j)+2 P_{p}^{*}(j, j) \lambda_{j}{ }^{2}\right)\right]^{+}, I_{t h}\right\}, \forall j=1, \ldots, N-n, \\
{\left[\frac{1}{\mu_{s} A_{s}(j, j)+\sum_{l=1}^{L}\left(\eta_{s_{l}} \sum_{i=1}^{N}\left|H_{s_{l}}(j, i)\right|^{2}\right)}-Q_{\tilde{z}}(j, j)\right]^{+},} \\
\forall j=N-n+1, \ldots, N .
\end{array}\right.
\end{aligned}
$$

We notice, here, that the optimal power involves directly the primary power and eigenmodes. Consequently, the SU power allocation is more sensitive tot eh PU channel variation than in the case of perfect SIC.

We adopt a Rayleigh fading channel in which the channel gains are complex Gaussian random variables with zero mean and unit variance. We choose $N=4$ antennas, and the rates expressed in bits per channel use (BPCU). We consider the same budget power at the PU and the SU transmitters, i.e., $P_{t o t, p}=P_{t o t, s}=P_{t o t}$. For simplicity, we assume that the relays amplification matrices are diagonal and equal and are given by: $\boldsymbol{W}=w \times \boldsymbol{I}_{\boldsymbol{N}}$ where $w$ is a positive scalar and $\boldsymbol{I}_{N}$ is the $\mathrm{N}$-dimension identity matrix. We also take an equal power budget at all the relays, i.e., $P_{R_{1}}=\cdot=P_{R_{L}}=P_{R}$. Note that the proposed scheme can be applied to any fixed amplification gain matrix. The optimization of $\boldsymbol{W}$ is left to a future extension of this work.

In Figure 2.a, we plot the PU and SU rates as a function of $P_{t o t}$ for $P_{R}=10 \mathrm{~dB}$ and $w=0.4$ with perfect SIC $(\alpha=0)$ and with various number of relays, $L=1,2,4$. We show that the space alignment technique allows the SU to achieve a free eigenmodes rate $R_{S}(F E)$, i.e. there is no tolerated interference from the PU, up to 0.5 BPCU for $L=1$ and 1.1 BPCU for $L=4$. However, this rate becomes zero when $P_{t o t}$ exceeds $17 \mathrm{~dB}$ for $L=1$ and becomes constant for $L>1$ since, in this regime, the PU is using most of the eigenmodes. 


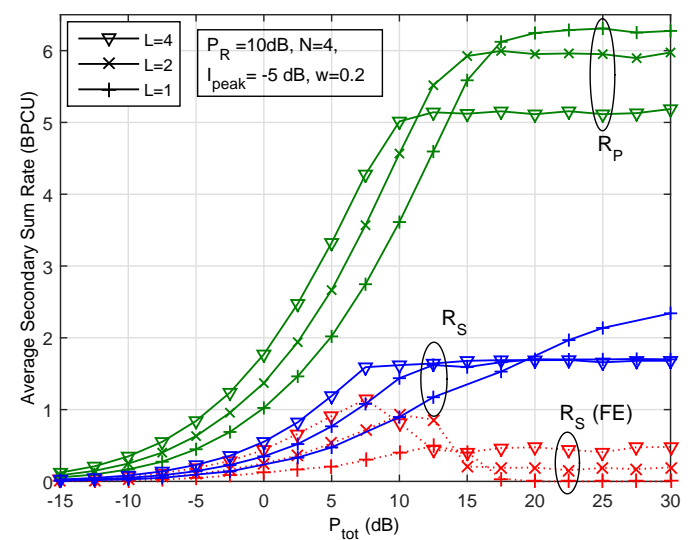

(a) Perfect SIC.

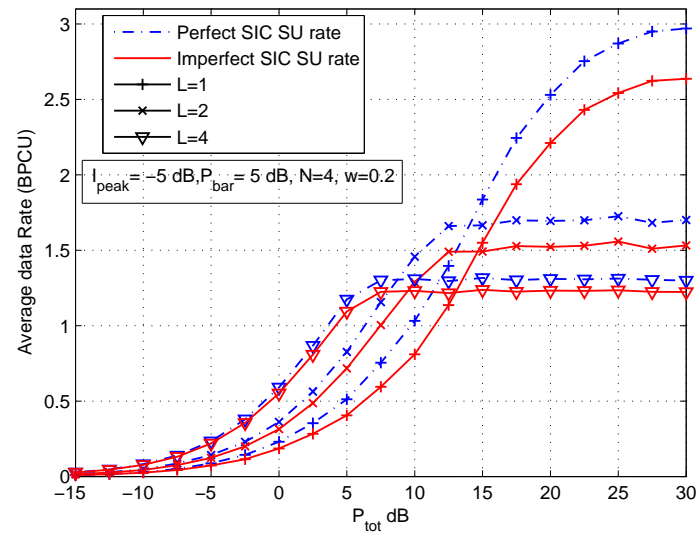

(b) Imperfect SIC.

Figure 2: PU and SU Rates versus $P_{\text {tot }}$.

We also show that at low values of $P_{t o t}$, increasing the number of relays enhances both PU and SU rates. In fact, in this range, the relays are not saturated, i.e. the relays constraints are not active. That is, adding more relays will further amplify the PU and SU signals and give better performances. However, as $P_{t o t}$ increases, the performances start to stagnate at a certain fixed levels due to the saturation of the relays. We also notice that this saturation level of the rates decrease when $L$ increases. In fact, since all the relays constraints are active, the more relays are available, the more constraints we are applying to the PU and SU transmit power. Hence, the power should satisfy a more strict constraint by respecting the lower constraint at each time. In average, the resulting performance is lower than the one with fewer relays. In order to study the SU rate loss between perfect and imperfect SIC, in Figure 2.b, the SU rate with perfect and imperfect SIC is presented for $P_{R}=10 \mathrm{~dB}$. We notice that as $L$ increases, the gap between perfect and imperfect SIC rates decreases from $17 \%$ for $L=1$ to $6 \%$ for $L=4$ for $P_{t o t}=20 \mathrm{~dB}$. This is explained by the fact that the PU power decrees with $L$ and hence from (24), the SU power of imperfect SIC increase with $L$ and the imperfect SIC rate becomes closer to the perfect SIC one.

Figure 3 shows the effect of the relay's power, $P_{R}$, on the PU and SU rates with different values of $L$. First, we notice

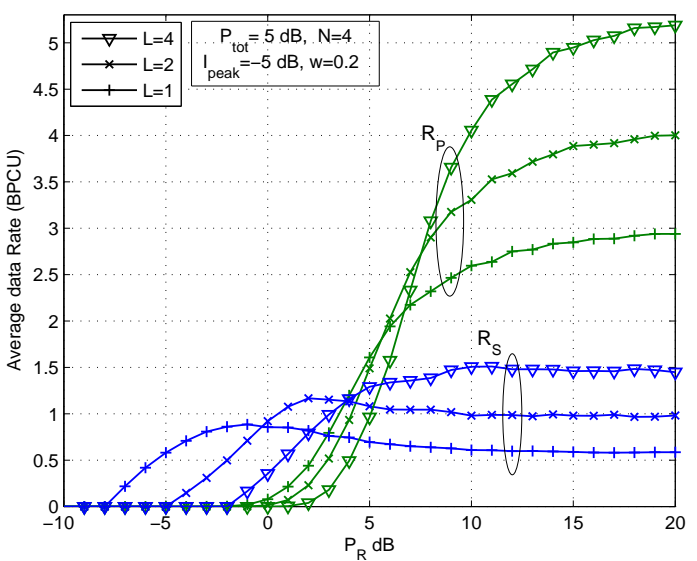

Figure 3: PU and SU Rates with perfect SIC versus $P_{R}$.

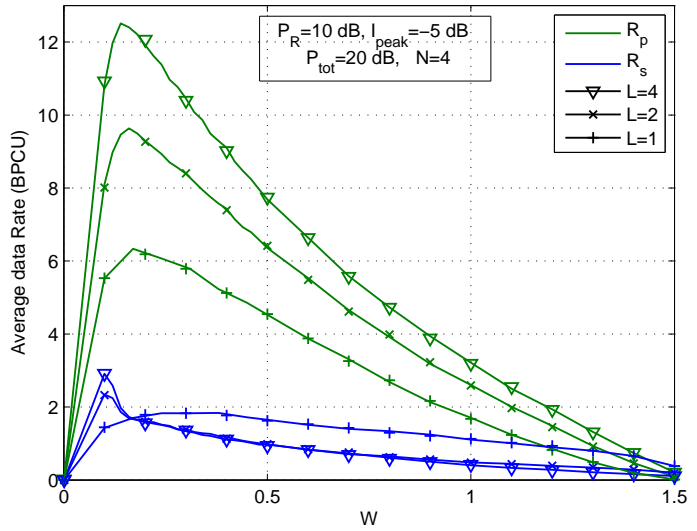

Figure 4: PU and SU rates with perfect SIC versus $w$.

that when $P_{R}$ is low, the SU reaches its maximum rate before starting to slightly decrease. Meanwhile, the primary rate is very reduced since its power is limited, in (9), by the low relay's power and the terms involving $I_{t h} \boldsymbol{H}_{s_{l}} \boldsymbol{H}_{s_{l}}{ }^{h}$ which is independent of $P_{R}$. Hence the optimal PU power, $P_{p}^{*}$, is limited and close to zero. Meanwhile, the SU power in (17) is limited by the relays power $P_{R}$, in addition, $\boldsymbol{H}_{\boldsymbol{p}_{l}} \boldsymbol{P}_{\boldsymbol{p}}^{*} \boldsymbol{H}_{\boldsymbol{p}_{l}}{ }^{h}$ which is already very low, consequently the power budgets of the relays are, in this regime, entirely dedicated to the SU. However, when $P_{R}$ becomes greater, the cognitive rate stagnates or decreases while the primary rate increases remarkably to the no cognition upper bound. Hence, the choice of $P_{R}$ is critical to the PU since the SU rate is almost the same.

In Figure 4, we highlight the effect of the relay amplification matrix gain of the relays $\boldsymbol{W}$ on PU and SU rates for different values of $L$. Recall that, we considered all the gain matrices to be equal to $\boldsymbol{W}=w \times \boldsymbol{I}_{\boldsymbol{N}}$, which is not necessarily the optimal choice but is a simple one to quantify the effect of this matrix on the system performance. We notice that the PU and SU rates reach a maximum for a particular value of $w$ before decreasing to zero as $w$ increases. The reason behind this rate shape is that increasing $w$ enhances the power as the relays constraints are not active. When reached, i.e., the values of $w$ are large, the transmit power should be small in order to respect the constraint and as $w$ increases further, the power 


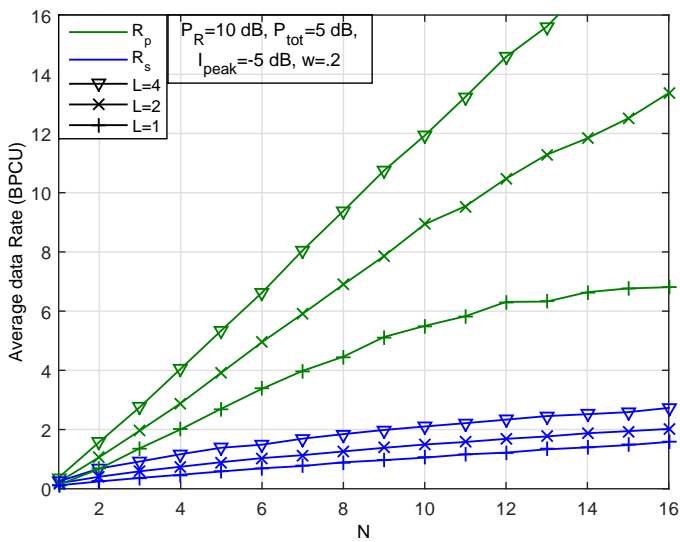

Figure 5: PU and SU rates with perfect SIC versus $N$.

should be near zero which applies for both PU and SU rates. Besides, the optimal $w$ giving the maximum rate is slightly different for PU and SU and can favor one over the other as shown in Figure 4. By measuring the maxima rate increase between $L=1$ and $L=4$, we find and increase of $97 \%$ for the PU and $105 \%$ for the SU. Meanwhile, we notice that this maximum is independent from the number of relays, in fact the optimal $w$ for $L=1$ is the same for $L=2$ and $L=3$ which means that finding the optimal $w$ is important since any additional relays to the system should adopt this values in order to give maximum performance.

In Figure 5, we study the effect of the number of antennas on the PU and SU rates with perfect SIC and different number of relays $L$. We first notice that the increasing slope of the PU and SU rates with $\mathrm{N}$ is almost linear except for the PU rate when $L=1$. However, increasing the number of relays enhances considerably both $\mathrm{PU}$ and SU rates, e.g. for $N=8$, the PU and SU rate increases by $110 \%$ and $106 \%$, respectively between $L=1$ and $L=4$.

\section{CONCLUSION}

In this paper, we proposed a simplified scheme to determine the optimal power allocation for the PU and SU users in an amplify-and-forward multi-relays network. The common destination performs a successive interference cancellation (SIC) technique to decode both signals. We have also derived the optimal power in different settings (perfect and imperfect SIC) in order to give upper and lower bounds of the cognitive rate. We highlighted the effect of the number of relays on the system's performances. We showed that increasing the number of relays enhances PU and SU rates at low power regime when the relays budget power is not attained. We also showed that, in the case of perfect SIC, the corresponding SU rate drops by at most $17 \%$. We also showed that the relays gain matrices considerably affect PU and SU rates and that the relay gain that maximizes the PU rate is slightly different that the one that maximizes the $\mathrm{SU}$ rate.

\section{ACKNOWLEDGEMENT}

This work was funded in part by a grant from King Abdulaziz City of Sciences and Technology (KACST).

\section{REFERENCES}

[1] "Spectrum policy task force," Federal Communications Commission, Tech. Rep. ET Docket no. 02-135, Nov. 2002.

[2] J. Mitola and G. Q. Maguire, "Cognitive radio: Making software radios more personal," IEEE. Personal Communications, vol. 6, no. 4, pp. 1318, Aug. 1999.

[3] T. Cover and A. EL Gamal, "Capacity theorems for the relay channel," IEEE Transactions on Information Theory, vol. 25, no. 5, pp. 572-584, 1979.

[4] B. Wang, J. Zhang, and A. Host-Madsen, "On the capacity of MIMO relay channels," IEEE Transactions on Information Theory, vol. 51, no. 1, pp. 29-43, 2005.

[5] L. Sboui, H. Ghazzai, Z. Rezki, and M.-S. Alouini, "Achievable rate of cognitive radio spectrum sharing MIMO channel with space alignment and interference temperature precoding," in Proc. of the IEEE International Conference on Communications (ICC'13), Budapest, Hungary, Budapest, Hungary, Jun. 2013, pp. 2656-2660.

[6] S. Perlaza, M. Debbah, S. Lasaulce, and J.-M. Chaufray, "Opportunistic interference alignment in MIMO interference channels," in Proc. of the 19th IEEE International Symposium on Personal, Indoor and Mobile Radio Communications (PIMRC'08), Cannes, France, Sep. 2008.

[7] X. Kang, Y.-C. Liang, and A. Nallanathan, "Optimal power allocation for fading channels in cognitive radio networks under transmit and interference power constraints," in Proc. of the IEEE International Conference on Communications (ICC'08), Beijing, China, May 2008, pp. $3568-3572$.

[8] M. Choi, J. Park, and S. Choi, "Simplified power allocation scheme for cognitive multi-node relay networks," IEEE Transactions on Wireless Communications, vol. 11, no. 6, pp. 2008-2012, 2012.

[9] J. Lee, H. Wang, J. G. Andrews, and D. Hong, "Outage probability of cognitive relay networks with interference constraints," IEEE Transactions on Wireless Communications, vol. 10, no. 2, pp. 390-395, 2011.

[10] M. Naeem, D. Lee, and U. Pareek, "An efficient multiple relay selection scheme for cognitive radio systems," in IEEE International Conference on Communications Workshops (ICC), Cape Town, South Africa, 2010, pp. $1-5$.

[11] L. Sboui, H. Ghazzai, Z. Rezki, and M.-S. Alouini, "On the throughput of a Relay-Assisted cognitive radio MIMO channel with space alignment," in 12th International Symposium and Workshops on Modeling and Optimization in Mobile, Ad Hoc and Wireless Networks (WiOpt '14), Hammamet, Tunisia, May 2014, pp. 317-323.

[12] I. Krikidis, "A svd-based location coding for cognitive radio in mimo uplink channels," IEEE Communications Letters, vol. 14, no. 10, pp. 912-914, 2010.

[13] L. Sboui, H. Ghazzai, Z. Rezki, and M.-S. Alouini, "Achievable rate of a cognitive MIMO multiple access channel with multi-secondary users," IEEE Communications Letters, vol. PP, no. 99, pp. 1-1, 2015.

[14] P. Popovski, H. Yomo, K. Nishimori, R. Di Taranto, and R. Prasad, "Opportunistic interference cancellation in cognitive radio systems," in Proc. of the 2nd IEEE International Symposium on New Frontiers in Dynamic Spectrum Access Networks (DySPAN'07), Dublin, Ireland, Apr. 2007, pp. 472-475.

[15] M. Qingyu, A. Osseiran, and J. Gan, "MIMO amplify-and-forward relaying: spatial gain and filter matrix design," in Proc. IEEE International Conference on Communications Workshops (ICC Workshops'08), Beijing, China, 2008.

[16] S. Haykin, "Cognitive radio: Brain-empowered wireless communications," IEEE Journal on Selected Areas in Communications, vol. 23, no. 2, pp. 201-220, Feb. 2005.

[17] S. Boyd and L. Vandenberghe, Convex Optimization. Cambridge University Press, 2004.

[18] L. Sboui, Z. Rezki, and M.-S. Alouini, "A unified framework for the ergodic capacity of spectrum sharing cognitive radio systems," IEEE Transactions on Wireless Communications, vol. 12, no. 2, pp. 877-887, Feb. 2013. 